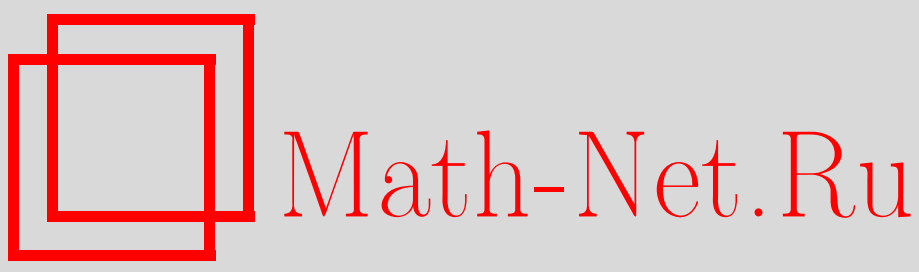

Д. Бертран, В. В. Зудилин, Производные зигелевых модулярных форм и показательные функции, Изв. РАН. Сер. матем., 2001, том 65, выпуск 4, 21-34

DOI: https://doi.org/10.4213/im345

Использование Общероссийского математического портала Math-Net.Ru подразумевает, что вы прочитали и согласны с пользовательским соглашением http://www . mathnet.ru/rus/agreement

Параметры загрузки:

IP: 54.157 .27 .8

26 апреля 2023 г., 18:22:30 
УДК 511.3

Д. Бертран, В.В. Зудилин

\title{
Производные зигелевых модулярных форм и показательные функции
}

\begin{abstract}
Доказывается, что дифференциальное поле, порожденное зигелевыми модулярными формами, и дифференциальное поле, порожденное экспонентами многочленов, линейно разделены над $\mathbb{C}$. Вместе с теоремой из нашей предыдущей работы [3] этот результат дает полное многомерное обобщение теоремы Малера о степени трансцендентности поля, порожденного показательной функцией и производными модулярной функции. Приводятся два доказательства нашего результата: чисто алгебраическое и аналитическое, но в основе обоих лежат аргументы дифференциальной алгебры, а также устойчивость дифференциального поля, порожденного рациональными и модулярными функциями, под действием симплектической группы.

Библиография: 10 наименований.
\end{abstract}

\section{§1. Введение и формулировка результатов}

В 1969 г. К. Малер [1] доказал, что для любой модулярной функции $f:\{\tau \in \mathbb{C}$ : $\operatorname{Im} \tau>0\} \rightarrow \mathbb{C}$, отличной от постоянной, и любого ненулевого комплексного числа $c$ пять функций

$$
\tau, q(\tau)=e^{c \tau}, f(\tau), f^{\prime}(\tau) \text { и } f^{\prime \prime}(\tau)
$$

алгебраически независимы над $\mathbb{C}$; здесь штрих обозначает дифференширование по $\tau$. Поскольку $f^{\prime \prime \prime}(\tau)$ рациональна над $\mathbb{C}\left(f(\tau), f^{\prime}(\tau), f^{\prime \prime}(\tau)\right)$, каждое из полей

$$
\begin{array}{cc}
\mathbb{C}\left(f(\tau), f^{\prime}(\tau), f^{\prime \prime}(\tau)\right), & \mathbb{C}\left(\tau, f(\tau), f^{\prime}(\tau), f^{\prime \prime}(\tau)\right), \\
\mathbb{C}\left(q(\tau), f(\tau), f^{\prime}(\tau), f^{\prime \prime}(\tau)\right), & \mathbb{C}\left(\tau, q(\tau), f(\tau), f^{\prime}(\tau), f^{\prime \prime}(\tau)\right)
\end{array}
$$

является дифференциально замкнутым, и соответствующие степени трансцендентности над $\mathbb{C}$ равны $3,4,4,5$. Результат Малера был распространен, с одной стороны, на случай общих автоморфных функций одной переменной К. Нишиокой в статье [2] и, с другой стороны, на случай зигелевых модулярных функций произвольной степени в нашей совместной статье [3], однако соответствуюший набор функций в [3] не содержит экспоненты. Цель настоящей работы - завершить обобшение работы [3] путем добавления показательных функций.

Для формулировки результатов мы воспользуемся обозначениями из [3]. Пусть $g$ - положительное целое (обозначающее степень или род) и $k$ - алгебраически замкнутое подполе $\mathbb{C}$.

Работа выполнена при частичной поддержке фонда INTAS и Российского фонда фундаментальных исследований (грант № IR-97-1904).

(C) Д. Бертран, В. В. Зудилин, 2001 
Положим:

$\mathfrak{H}_{g}$ - верхнее полупространство Зигеля степени $g ; \mathbb{Q}$-векторная группа $Z_{g}$, порожденная симметрическими матрицами порядка $g$, имеет размерность

$$
n:=\frac{g(g+1)}{2},
$$

при этом $\mathfrak{H}_{g}$ открыто в $Z_{g}(\mathbb{C})$;

$\boldsymbol{\tau}=\left(\tau_{j l}\right)_{1 \leqslant j \leqslant l \leqslant g}$ - обшая точка в $\mathfrak{H}_{g}$, так что $k(2 \pi i \tau)$ можно рассматривать как поле рациональных функций на $Z_{g} / k$;

$$
\delta=\left\{\delta_{j l}, 1 \leqslant j \leqslant l \leqslant g\right\}, \text { где }
$$

$$
\delta_{j l}=\frac{1}{2 \pi i} \frac{\partial}{\partial \tau_{j l}}, \quad 1 \leqslant j<l \leqslant g, \quad \delta_{j j}=\frac{1}{\pi i} \frac{\partial}{\partial \tau_{j j}}, \quad 1 \leqslant j \leqslant g
$$

эти $n$ частных производных (дифференцирований) образуют $k(2 \pi i \tau)$-базис пространства $\operatorname{Der}(k(2 \pi i \tau) / k)$;

$\Gamma$ - конгруэнщ-подгруппа симплектической группы $S p_{2 g}(\mathbb{Z})$ (или, что то же самое, подгруппа конечного индекса в случае $g>1$ );

$K:=K(\Gamma, k)$-поле модулярных функций относительно конгруэнц-подгрупшы $\Gamma$; как известно, это поле является конечно порожденным расширением $k$, имеющим степень трансцендентности

$$
\operatorname{tr} \operatorname{deg}_{k} K=\frac{g(g+1)}{2}=n ;
$$

при $g>1$ поле $K \otimes_{k} \mathbb{C}$ отождествляется с полем мероморфных на $\mathfrak{H}_{g}$ функций, инвариантных под действием групшы $\Gamma$;

$M:=M(\Gamma, k)-\boldsymbol{\delta}$-дифференциальное поле, порожденное $K$, т.е. поле, порожденное над $k$ всеми частными $\delta_{j l}$-производными любого порядка элементов из $K$.

В [3, теорема 1] мы доказали, что $\delta$-дифференциальное поле $M$ является конечным расширением поля, порожденного $\delta$-частными производными элементов из $K$ порядка не вьше 2 , и имеет конечную степень трансцендентности над $k$, равную

$$
\operatorname{tr} \operatorname{deg}_{k} M=\operatorname{dim} S p_{2 g}=2 g^{2}+g ;
$$

более того, поля $M u \mathbb{C}(\boldsymbol{\tau})$ линейно разделены над $k$, поэтому

$$
\operatorname{tr} \operatorname{deg}_{k} M(\boldsymbol{\tau})=\operatorname{dim} S p_{2 g}+n=\frac{1}{2} g(5 g+3) .
$$

Мы обобщаем эту теорему следующим образом.

Теорема 1. Пусть $M-\delta$-дифференциальное поле, отвечающее полю модулярных функиий $K$, и пусть $c$ - произвольное ненулевое комплексное число. Тогда показательнье функции $e^{c \tau_{j l}}, 1 \leqslant j \leqslant l \leqslant g$, алгебраччески независимы над $M(\boldsymbol{\tau})$, поэтому

$$
\operatorname{tr} \operatorname{deg}_{k} M\left(\boldsymbol{\tau}, e^{c \boldsymbol{\tau}}\right)=\operatorname{dim} S p_{2 g}+2 n=3 g^{2}+2 g .
$$


Настояшая работа устроена следуюшим образом. $\S 2$ содержит некоторые вспомогательные утверждения о действии группы $\Gamma$ на $\mathfrak{H}_{g}$ и $Z_{g}$. В $\S 3,4$ мы приводим два независимых доказательства теоремы 1 . Более точно, доказательство из $\S 3$ основано на теореме Акса (функциональной версии гипотезы Шенуэла) и имеет чисто алгебраическую природу, в то время как доказательство из $\S 4$ использует более простую версию теоремы Акса (а именно, доказанньй Колчиным мультипликативный аналог теоремы Островского) и аналитические рассуждения. На самом деле, в $§ 4$ мы доказываем следуюшее усиление теоремы 1.

Теорема 2. В обозначениях теоремы 1 пусть $\left\{Q_{1}, \ldots, Q_{N}\right\}$ - произвольное множество многочленов от переменных $\tau=\left(\tau_{j l}\right)_{1 \leqslant j \leqslant l \leqslant g}$ с комплексными коэффициентами и без свободных членов. Предположсим, что многочлены $Q_{1}, \ldots, Q_{N}$ линейно независимы над $\mathbb{Q}$. Тогда функции $e^{Q_{1}(\boldsymbol{\tau})}, \ldots, e^{Q_{N}(\boldsymbol{\tau})}$ алгебраически независимы над $M(\tau)$.

Наконец, $\S 5$ посвящен изучению модулярных тэта-констант; особое внимание уделяется роду 2 , в котором мы приводим явные выражения для разложений Фурье некоторых логарифмических производных тэта-констант (т.е. разложений в терминах показательных функций $e^{c \tau_{j l}}$ при $c=\pi i$ ).

ЗАмЕчАниЕ 1. Несмотря на то, что наши рассуждения следуют идеям доказательства из статьи [2], представленные здесь как алгебраический, так и аналитический методы добавления показательных функций к дифференциальному полю, порожденному модулярньги формами, являются новыми даже в случае рода 1 . Второй автор использовал аналогичные аргументы в другой работе [4] для добавления показательной функции к дифференциальному полю, порожденному так называемым (квантовым) спариванием Юкавы.

\section{§2. Вспомогательные утверждения}

Как отмечено в [3], достаточно доказать наши теоремы в случае полной модулярной групшы

$$
\Gamma=S p_{2 g}(\mathbb{Z})
$$

кроме того, не ограничивая обшности можно считать, что поле констант совпадает с полем комплексных чисел:

$$
k=\mathbb{C} .
$$

В дальнейшем мы будем считать эти требования выполненными.

Точно так же, как и в [1], [2], следуюшее утверждение играет ключевую роль в наших доказательствах. Вместе с конечностью степени трансцендентности $M$ над $\mathbb{C}$ (см. формулу (1) из 11 и далее $\S 3$ или, альтернативно, с алгебраической независимостью $\tau_{j l}$ над $M$ (см. формулу (2) из $\S 1$ и далее $\S 4$ ) нам, в действительности, понадобится всего лишь одно свойство дифференциального поля $M(\tau)^{1}$. Напомним, что группа Г представляет дробно-линейное действие (слева) на верхнем полупространстве Зигеля $\mathfrak{H}_{g}$ :

$$
(\gamma, \boldsymbol{\tau}) \mapsto \gamma \cdot \boldsymbol{\tau}:=(a \boldsymbol{\tau}+b)(c \boldsymbol{\tau}+d)^{-1} \quad \text { для всех } \quad\left(\gamma=\left(\begin{array}{cc}
a & b \\
c & d
\end{array}\right), \boldsymbol{\tau}\right) \in \Gamma \times \mathfrak{H}_{g},
$$

\footnotetext{
${ }^{1}$ См. замечания 2,3 и 4 в конце $\S 4$, в которых приводится более детальное сравнение методов двух наших доказательств.
} 


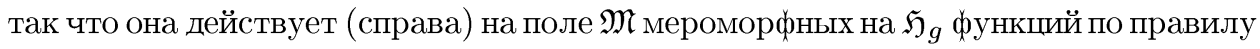

$$
(\gamma, f) \mapsto \gamma \cdot f: \boldsymbol{\tau} \mapsto(\gamma \cdot f)(\boldsymbol{\tau}):=f(\gamma \cdot \boldsymbol{\tau}) \quad \text { для всех }(\gamma, f) \in \Gamma \times \mathfrak{M} .
$$

Подполе $M$ поля $\mathfrak{M}$ не является устойчивым под действием $\Gamma$, но имеет место следующее утверждение.

ЛЕмма 1. Поле $M(\tau)$ устойчиво под действием әруппь Г.

ДоказАТельство. Для каждого $m=0,1, \ldots, \infty$ так же, как и в $[3, \S 4]$, обозначим через $K^{(m)}$ поле, порожденное над $\mathbb{C}$ всеми $\boldsymbol{\delta}$-производными порядка не выше $m$ всех элементов из $K$. Тогда $K^{(0)}=K \subset K^{(1)} \subset \cdots \subset K^{(\infty)}=M$. Индукцией по $m$ покажем устойчивость $K^{(m)}(\boldsymbol{\tau})$ под действием Г. Определение поля $K$ делает это утверждение очевидным при $m=0$ (а также при $m=1$ согласно $[3, \S 5$, формула (4)]). В случае произвольного $m$ рассмотрим произвольный элемент $f$ поля $K^{(m)}(\boldsymbol{\tau})$ и произвольный $\gamma \in \Gamma$; напомним, что дифференщиал в точке $\tau$ автоморфизма $\gamma=\left(\begin{array}{ll}a & b \\ c & d\end{array}\right)$ группы $\mathfrak{H}_{g}$ задается формулой

$$
\mathrm{d}_{\boldsymbol{\tau}} \gamma={ }^{t}(c \boldsymbol{\tau}+d)^{-1} \mathrm{~d} \boldsymbol{\tau}(c \boldsymbol{\tau}+d)^{-1}
$$

Поскольку

$$
\mathrm{d}_{\boldsymbol{\tau}}(\gamma \cdot f)=\mathrm{d}_{\gamma \cdot \boldsymbol{\tau}} f \circ \mathrm{d}_{\boldsymbol{\tau}} \gamma, \quad\left(\mathrm{d}_{\boldsymbol{\tau}} \gamma\right)^{-1}=\mathrm{d}_{\gamma \cdot \boldsymbol{\tau}}\left(\gamma^{-1}\right)
$$

и $g=\gamma \cdot f$ лежит в $K^{(m)}(\boldsymbol{\tau})$ по индуктивному предположению, мы заключаем, что каждая компонента $\gamma \cdot\left(\delta_{j l} f\right)$ дифференциала $\mathrm{d}_{\gamma \cdot \tau} f$ принадлежит полю, порожденному над $K^{(m)}(\boldsymbol{\tau})$ всеми $\boldsymbol{\delta}$-производными первого порядка всех элементов $g$ из $K^{(m)}(\boldsymbol{\tau})$. Но последнее поле совпадает с $K^{(m+1)}(\boldsymbol{\tau})$, и поэтому $K^{(m+1)}(\boldsymbol{\tau})$ является устойчивым под действием $\gamma$. Лемма доказана.

Следуюшие утверждения этого параграф̆а являются упражнениями по коммутативной алгебре.

Лемма 2. Многочлен $\operatorname{det} \tau \in \mathbb{C}\left[\tau_{j l}, \quad 1 \leqslant j \leqslant l \leqslant g\right]$ неприводим.

ДокаЗАТЕльство (ср. с упражнением 3 из [5, §30], в котором рассматривается случай произвольной матрицы). Достаточно показать неприводимость многочлена

$$
P_{g}\left(\tau_{1}, \ldots, \tau_{g}\right)=\operatorname{det} \tau^{*}, \quad \text { где } \tau_{j l}^{*}= \begin{cases}\tau_{j}, & \text { если } j=l, \\ 1, & \text { если } j=l+1 \text { или } l=j+1 \\ 0 & \text { иначе. }\end{cases}
$$

Действительно, при такой специализации общая степень $g$ многочлена $\operatorname{det} \tau$ не убывает. Мы докажем наше утверждение индукцией по $g$, начиная с многочленов $P_{1}\left(\tau_{1}\right)=\tau_{1}$ и $P_{2}\left(\tau_{1}, \tau_{2}\right)=\tau_{1} \tau_{2}-1$, неприводимость которых очевидна. Раскладывая $\operatorname{det} \tau^{*}$ по последнему столбцу (или строке), получаем

$$
P_{g}\left(\tau_{1}, \ldots, \tau_{g}\right)=\tau_{g} P_{g-1}\left(\tau_{1}, \ldots, \tau_{g-1}\right)-P_{g-2}\left(\tau_{1}, \ldots, \tau_{g-2}\right) .
$$

Предположим теперь, что многочлен $P_{g}$ приводим. Применяя лемму Гаусса к кольцу $\mathbb{C}\left[\tau_{1}, \ldots, \tau_{g-1}\right]\left[\tau_{g}\right]$, согласно индукционному предположению заключаем, что многочлены $P_{g-1}$ и $P_{g-2}$ делят друг друга, а это, очевидно, невозможно. Полученное противоречие завершает доказательство. 
СлЕДСТВИЕ. Для любого $m \in \mathbb{Z}$ многочлен $\operatorname{det}\left(\boldsymbol{\tau}-m \mathbf{1}_{g}\right) \in \mathbb{C}[\boldsymbol{\tau}]$ неприводим. В частности, для любой пары различных чельх $m_{1}, m_{2}$ многочлены $\operatorname{det}\left(\boldsymbol{\tau}-m_{1} \mathbf{1}_{g}\right) u \operatorname{det}\left(\boldsymbol{\tau}-m_{2} \mathbf{1}_{g}\right)$ взаимно простыв в $\mathbb{C}[\boldsymbol{\tau}]$.

ДоКАЗАТЕЛЬСТво. Полагая $\boldsymbol{\tau}^{\prime}=\boldsymbol{\tau}-m \mathbf{1}_{g}$, мы сводим первое утверждение к лемме 2 . Второе утверждение следует из невырожденности $\operatorname{det}\left(\boldsymbol{\tau}-m_{2} \mathbf{1}_{g}\right)$ в точке $\tau=m_{1} \mathbf{1}_{g}$.

В следующих утверждениях мы рассматриваем левое действие групшы $Г$ на полном векторном пространстве $Z_{g}$ симметрических матриц (расширяюшее указанное вьше действие на $\left.\mathfrak{H}_{g}\right)$ и соответствующее правое действие $\Gamma$ на поле $\mathbb{C}(\boldsymbol{\tau})$ рациональных функций на $Z_{g} / \mathbb{C}$. В частности, нам понадобятся элементы Г вида

$$
\gamma_{m}=\left(\begin{array}{cc}
\mathbf{0}_{g} & -\mathbf{1}_{g} \\
\mathbf{1}_{g} & -m \mathbf{1}_{g}
\end{array}\right) \in S p_{2 g}(\mathbb{Z}), \quad \gamma_{m}(\boldsymbol{\tau}):=\gamma_{m} \cdot \tau=-\left(\boldsymbol{\tau}-m \mathbf{1}_{g}\right)^{-1}
$$

где $m$ - произвольное рациональное целое. Для каждого такого $m$ положим

$$
\mathscr{D}_{m}=\left\{\boldsymbol{\tau} \in Z_{g}: \operatorname{det}\left(\boldsymbol{\tau}-m \mathbf{1}_{g}\right)=0\right\} .
$$

Согласно следствию из леммы 2 множество $\mathscr{D}_{m}$ является неприводимым дивизором аффинного пространства $Z_{g}$, а дивизоры $\mathscr{D}_{m_{1}}, \mathscr{D}_{m_{2}}$ различны для $m_{1} \neq m_{2}$.

Лемма 3. Пусть $P \in \mathbb{C}[\boldsymbol{\tau}]$ - ненулевой однородный многочлен и $m \in \mathbb{Z}$. Положим $R=\gamma_{m} \cdot P$, т.е. $R(\boldsymbol{\tau})=P\left(-\left(\boldsymbol{\tau}-m \mathbf{1}_{g}\right)^{-1}\right)$. Тогда $R$ является ненулевой рациональной функцией на $Z_{g}$, чей полярный дивизор совпадает $c(\operatorname{deg} P) \cdot \mathscr{D}_{m}$.

ДокАЗАТЕЛЬСТво. Сдвигая при необходимости параметр $m$, мы можем считать $m=0$, так что

$$
\gamma_{0}(\boldsymbol{\tau})=-\boldsymbol{\tau}^{-1}=\left(\frac{T_{j l}}{\operatorname{det} \boldsymbol{\tau}}\right)_{1 \leqslant j, l \leqslant g}
$$

где $(-1)^{j+l+1} T_{j l}$ обозначает определитель $(j, l)$-минора матрицы $\tau$. В частности, выражения $T_{j l}=T_{l j}$ являются однородными многочленами степени $g-1$ в кольце $\mathbb{C}[\tau]$. Поэтому

$$
R(\boldsymbol{\tau})=P\left(-\boldsymbol{\tau}^{-1}\right)=P\left(\frac{T_{j l}}{\operatorname{det} \boldsymbol{\tau}}\right)=\frac{N(\boldsymbol{\tau})}{(\operatorname{det} \boldsymbol{\tau})^{\operatorname{deg} P}},
$$

где $N(\boldsymbol{\tau})=P\left(T_{j l}\right)$ - либо нулевой, либо однородньй многочлен в $\mathbb{C}[\boldsymbol{\tau}]$ степени, равной $(g-1) \operatorname{deg} P$. Поскольку $P \neq 0$ по условию, невырожденность $R$, а значит, и $N$ очевидна. Следовательно, $N$ является однородным многочленом степени $(g-1) \operatorname{deg} P<\operatorname{deg}(\operatorname{det} \boldsymbol{\tau})^{\operatorname{deg} P}$. Ввиду невырожденности дивизора $\mathscr{D}_{0}=\left\{\boldsymbol{\tau} \in Z_{g}\right.$ : $\operatorname{det} \tau=0\}$ мы заключаем, что полярный дивизор рациональной функции $R$ в $Z_{g}$ является целым положительным кратным $\nu \mathscr{D}_{0}$ дивизора $\mathscr{D}_{0}$. Иными словами, $R=$ $\tilde{N} / \tilde{D}$, где $\tilde{D}=(\operatorname{det} \tau)^{\nu}$ для некоторого целого $\nu \geqslant 1$ и $\tilde{N}$ взаимно прост с $\tilde{D}$ в кольце $\mathbb{C}[\tau]$; более того, $\nu \leqslant \operatorname{deg} P$ и $\operatorname{deg} \tilde{N}<\operatorname{deg} \tilde{D}=g \nu$. Это завершает доказательство в случае $\operatorname{deg} P=1$ и сводит обший случай к проверке равенства $\nu=\operatorname{deg} P$. 
Заметим, что случай $\operatorname{deg} P=1$ также влечет тот факт, что прообраз $\gamma_{0}^{-1}(\mathscr{H})$ аффинной гиперплоскости $\mathscr{H} \subset Z_{g}$ под действием автоморфизма $\gamma_{0}$ из $Z_{g}$ является гиперповерхностью степени $g$ в $Z_{g}$. Таким образом, образ $\gamma_{0}(\mathscr{L})$ аффинной прямой $\mathscr{L}$ в $Z_{g}$ является кривой степени $g$.

Далее, согласно $\operatorname{deg} \tilde{N}<\operatorname{deg} \tilde{D}$ степень $g \nu$ полярного дивизора $\nu \mathscr{D}_{0}$ рациональной функции $R$ может быть вычислена следующим образом. Пусть $\alpha-$ точка в $\mathbb{C}$ и $\mathscr{L}$ - прямая в аффинном пространстве $Z_{g}$. Тогда уравнение $R(\boldsymbol{\tau})=\alpha$ задает в $Z_{g}$ неприводимый и приведенный дивизор $D_{\alpha}$ степени $g \nu($ с уравнением $\tilde{N}-\alpha \tilde{D}=0)$, так что $D_{\alpha}$ пересекает прямую $\mathscr{L}$ в $g \nu$ простых точках. Поскольку $\gamma_{0}$ является как локальным, так и глобальным изоморфизмом $Z_{g}$, образы $\gamma_{0}\left(D_{\alpha}\right)$ и $\gamma_{0}(\mathscr{L})$ также пересекаются в $g \nu$ простых точках, именно $\gamma_{0}\left(D_{\alpha}\right) \cap \gamma_{0}(\mathscr{L})=\gamma_{0}\left(D_{\alpha} \cap \mathscr{L}\right)$. Но из представления $R=\gamma_{0} \cdot P$ следует, что

$$
\gamma_{0}\left(D_{\alpha}\right)=\left\{\boldsymbol{\tau}^{\prime}=\gamma_{0}(\boldsymbol{\tau}): R(\boldsymbol{\tau})=\alpha\right\}=\left\{\boldsymbol{\tau}^{\prime} \in Z_{g}: P\left(\boldsymbol{\tau}^{\prime}\right)=\alpha\right\}
$$

является дивизором степени $\operatorname{deg} P$, в то время как $\gamma_{0}(\mathscr{L})$ является кривой степени $g$. Кроме того, они пересекаются (в виду произвольности выбора $\alpha \in \mathbb{C}$ и $\mathscr{L}$ в $Z_{g}$ ) на конечном расстоянии и (как мы видели) однократно. Поэтому из теоремы Безу мы заключаем, что множество $\gamma_{0}\left(D_{\alpha}\right) \cap \gamma_{0}(\mathscr{L})$ состоит из $g \operatorname{deg} P$ различных точек. Следовательно, $g \nu=g \operatorname{deg} P$, откуда $\nu=\operatorname{deg} P$, что и требовалось доказать.

СлеДСТвИЕ. Пусть $t-$ положительное целое, $P_{0}, \ldots, P_{t} \in \mathbb{C}[\tau]-($ не обязательно однородные) ненулевые многочлены без постоянных членов и $Q$ произвольный ненулевой многочлен в $\mathbb{C}[\tau]$. Для каждого $m=0, \ldots, t$ положим $R_{m}(\boldsymbol{\tau})=P_{m}\left(-\left(\boldsymbol{\tau}-m \mathbf{1}_{g}\right)^{-1}\right)$. Тогда рачиональные функции $R_{0}, \ldots, R_{t}$ u $Q$ линейно независимы над $\mathbb{C}$.

ДокАЗАТЕльСТво. Прежде всего, заметим, что любое семейство ненулевых рациональных функций на $Z_{g}$, полярные дивизоры которых попарно различны, линейно независимо над $\mathbb{C}$. Здесь “попарно различны" означает "различны либо как множества, либо входят с разными кратностями", при этом тривиальный дивизор 0 (т.е. пустое множество) также может быть элементом этого семейства. Приведенное замечание очевидным образом следует из единственности разложения на множители в $\mathbb{C}[\boldsymbol{\tau}]$. Рассматривая теперь каждую ненулевую однородную компоненту $P_{m i}$ степени $i$ (где $i \in I_{m} \subset\left\{1, \ldots, \operatorname{deg} P_{m}\right\}$ ) каждого многочлена $P_{m}$ $(0 \leqslant m \leqslant t)$ из условия, согласно лемме 3 и следствию из леммы 2 мы получаем семейство ненулевых рациональных функций $P_{m i}\left(-\left(\boldsymbol{\tau}-m \mathbf{1}_{g}\right)^{-1}\right)$, которые вместе с многочленом $Q(\tau)$ допускают попарно различные полярные дивизоры, а именно $i \mathscr{D}_{m}\left(m=0, \ldots, t, i \in I_{m}\right)$ и 0 . Следовательно, это семейство линейно независимо над $\mathbb{C}$, и это же свойство вьполнено для исходного набора $R_{1}, \ldots, R_{t}, Q$.

\section{§3. Алгебраическое доказательство теоремы 1}

Основньм инструментом в этом доказательстве служит следующий результат Дж. Акса [6, теорема 4], являюшийся сильной функциональной версией гипотезы Шенуэла.

ПРЕДЛОЖЕНИЕ 1 (теорема Акса). Пусть $F \supseteq E \supseteq k \supseteq \mathbb{Q}-$ последовательность полей, $\Delta$ - множество дифференцирований поля $F$ таких, что 
для всех $\delta \in \Delta$ выполнено $\delta E \subseteq E, u \bigcap_{\delta \in \Delta} \operatorname{ker} \delta=k$. Пусть әлементь $y_{1}, \ldots, y_{s}, z_{1}, \ldots, z_{s} \in F^{*}$ таковы, что:

а) $\delta z_{r} / z_{r}-\delta y_{r} \in E$ для всех $\delta \in \Delta, r=1, \ldots, s$;

б) любое нетривиальное произведение степеней $z_{1}, \ldots, z_{s}$ не является алгебраичным над $E$.

Тогда

$$
\operatorname{tr} \operatorname{deg}_{E} E\left(y_{1}, \ldots, y_{s}, z_{1}, \ldots, z_{s}\right) \geqslant s .
$$

Предположим, что функции $e^{c \tau_{j l}}, 1 \leqslant j \leqslant l \leqslant g$, алгебраически зависимы над $M(\tau)$. Согласно лемме 1 это предположение означает, что для всякого автоморфизма $\gamma \in \Gamma$ функции $e^{c \tau_{j l}^{\prime}}, 1 \leqslant j \leqslant l \leqslant g$, где $\left(\tau_{j l}^{\prime}\right)=\gamma\left(\tau_{j l}\right)$, также алгебраически зависимы над $M(\boldsymbol{\tau})$. Поэтому, полагая $\boldsymbol{\tau}^{(m)}=\left(\tau_{j l}^{(m)}\right)=\gamma_{m}(\boldsymbol{\tau})$, $m=0,1, \ldots, t$, для любого набора $\gamma_{0}, \ldots, \gamma_{t} \in \Gamma$, мы получаем оценку сверху

$$
\begin{aligned}
\operatorname{tr} \operatorname{deg}_{M(\boldsymbol{\tau})} M\left(\boldsymbol{\tau}, e^{c \boldsymbol{\tau}^{(0)}}, e^{c \boldsymbol{\tau}^{(1)}}, \ldots, e^{c \boldsymbol{\tau}^{(t)}}\right) & \leqslant \#\left\{\boldsymbol{\tau}^{(m)}\right\}_{m=0,1, \ldots, t}-(t+1) \\
& =(t+1) n-(t+1) .
\end{aligned}
$$

Обозначим теперь

$$
\begin{gathered}
k=\mathbb{C}, \quad E=M, \quad F=M\left(\boldsymbol{\tau}, e^{c \boldsymbol{\tau}^{(0)}}, e^{c \boldsymbol{\tau}^{(1)}}, \ldots, e^{c \boldsymbol{\tau}^{(t)}}\right), \quad \Delta=\left\{\delta_{j l}\right\}, \\
\left\{y_{r}\right\}=\left\{c \tau_{j l}^{(m)}\right\} \quad \text { и } z_{r}=e^{y_{r}}, \quad r=1, \ldots, s
\end{gathered}
$$

с $s=(t+1) n$, в предложении 1 и допустим, что условие б) выполнено. Условие а) предложения 1 выполнено автоматически, так как $\delta z_{r} / z_{r}-\delta y_{r}=0$ для всех дифференцирований $\delta \in \Delta$ и любых $r=1, \ldots, s$; включение $\delta E \subseteq E$ следует из определения поля $M$. Тогда согласно теореме Акса

$$
\operatorname{tr} \operatorname{deg}_{M} M\left(y_{1}, \ldots, y_{s}, z_{1}, \ldots, z_{s}\right) \geqslant s, \quad \text { где } s=(t+1) n
$$

Вспоминая, что $M\left(y_{1}, \ldots, y_{s}\right)=M(c \tau)=M(\tau)$ и величина $\operatorname{tr} \operatorname{deg}_{M} M(\tau)$ конечна (более точно, равна $n$ ), мы получем оценку снизу

$$
\begin{aligned}
\operatorname{tr} \operatorname{deg}_{M(\boldsymbol{\tau})} M\left(\boldsymbol{\tau}, e^{c \boldsymbol{\tau}^{(0)}}, e^{c \boldsymbol{\tau}^{(1)}}, \ldots, e^{c \boldsymbol{\tau}^{(t)}}\right) & =\operatorname{tr} \operatorname{deg}_{M(\boldsymbol{\tau})} M\left(y_{1}, \ldots, y_{s}, z_{1}, \ldots, z_{s}\right) \\
& \geqslant s-\operatorname{tr} \operatorname{deg}_{M} M(\boldsymbol{\tau})=(t+1) n-n
\end{aligned}
$$

противоречащую оценке сверху для достаточно большого $t$ (именно, для $t \geqslant n$ ).

Таким образом, достаточно предъявить набор $\gamma_{0}, \gamma_{1}, \ldots, \gamma_{t} \in \Gamma=S p_{2 g}(\mathbb{Z})$, для которого выполнено условие б) теоремы Акса. Как и в доказательстве из [2], выберем

$$
\gamma_{m}=\left(\begin{array}{cc}
\mathbf{0}_{g} & -\mathbf{1}_{g} \\
\mathbf{1}_{g} & -m \mathbf{1}_{g}
\end{array}\right) \in S p_{2 g}(\mathbb{Z}), \quad m=0,1, \ldots, t .
$$

Обозначим, как выше,

$$
\boldsymbol{\tau}^{(m)}=\left(\tau_{j l}^{(m)}\right)=\gamma_{m}(\boldsymbol{\tau})=-\left(\boldsymbol{\tau}-m \mathbf{1}_{g}\right)^{-1}
$$


(т.е. $\tau_{j l}^{(m)}=\gamma_{m} \cdot \tau_{j l}$ в обозначениях $\left.\S 2\right)$ и предположим, что условие б) предложения 1 не выполнено для соответствуюшего выбора $\left\{y_{r}\right\}$ и $\left\{z_{r}=e^{y_{r}}\right\}$. Тогда существует нетривиальное множество $\left\{C_{j l}^{(m)}\right\} \in \mathbb{Z}^{n t}$ такое, что рациональная функция

$$
R(\boldsymbol{\tau})=\sum_{\substack{1 \leqslant j \leqslant l \leqslant g \\ 1 \leqslant m \leqslant t}} C_{j l}^{(m)} c \tau_{j l}^{(m)}
$$

удовлетворяет включению

$$
e^{R(\boldsymbol{\tau})} \in M^{\mathrm{alg}},
$$

где через $M^{\text {alg }}$ обозначено алгебраическое замыкание поля $M$. Вычисляя логарифмические производные и пользуясь дифференщиальной замкнутостью $M$, а значит, и $M^{\mathrm{alg}}$, мы приходим к соотношениям

$$
\frac{\partial R}{\partial \tau_{j l}}(\boldsymbol{\tau}) \in M^{\text {alg }} \quad \text { для всех } \quad 1 \leqslant j \leqslant l \leqslant g .
$$

Но поля $M^{\mathrm{alg}}$ и $\mathbb{C}(\boldsymbol{\tau})$ линейно разделены над $\mathbb{C}$, поскольку $M$ и $\mathbb{C}(\boldsymbol{\tau})$ линейно разделены над $\mathbb{C}($ см. формулу $(2)$ из $\S 1)$, а $\mathbb{C}(\boldsymbol{\tau})$ является чисто трансцендентньгм расширением поля $\mathbb{C}$, так что из полученных соотношений следует, что

$$
\frac{\partial R}{\partial \tau_{j l}}(\boldsymbol{\tau}) \in \mathbb{C} \quad \text { для всех } 1 \leqslant j \leqslant l \leqslant g .
$$

Следовательно, для некоторого многочлена $Q$ (степени не вьше 1) вьполнено

$$
R=Q \in \mathbb{C}[\tau] .
$$

Рассмотрим теперь $t$ многочленов

$$
P_{m}(\boldsymbol{\tau})=\sum_{1 \leqslant j \leqslant l \leqslant g} C_{j l}^{(m)} c \tau_{j l}, \quad m=0,1, \ldots, t .
$$

Для каждого $m$ образ $R_{m}=\gamma_{m} \cdot P_{m}$ многочлена $P_{m}$ под действием $\gamma_{m} \in \Gamma$ есть рациональная функция

$$
R_{m}(\boldsymbol{\tau})=P_{m}\left(-\left(\boldsymbol{\tau}-m \mathbf{1}_{g}\right)^{-1}\right)=\sum_{1 \leqslant j \leqslant l \leqslant g} C_{j l}^{(m)} c \tau_{j l}^{(m)}, \quad m=0,1, \ldots, t
$$

поскольку действие $\Gamma$ на $\mathbb{C}(\boldsymbol{\tau})$ является $\mathbb{C}$-линейным. Значит, $R=\sum_{0 \leqslant m \leqslant t} R_{m}$, и мы в итоге приходим к соотношению

$$
\sum_{0 \leqslant m \leqslant t} R_{m}=Q
$$

Многочлен $Q$ может тождественно быть равным нулю, но числовое множество $\left\{C_{j l}^{(m)} c\right\} \in \mathbb{C}^{n t}$ нетривиально и, значит, хотя бы один из многочленов $P_{m}$, $m=0, \ldots, t$, ненулевой. Эти многочлены являются линейными формами, в частности не содержат свободные члены. Поэтому последнее соотношение противоречит следствию из леммы 3 , и это противречие завершает доказательство теоремы 1. 


\section{§4. Аналитическое доказательство теоремы 2}

4.1. Новое доказательство теоремы 1 в случае $g=1$. В этом случае мы можем обойтись без дифференциальной алгебры. Напомним, что степень трансцендентности поля $M$ над $\mathbb{C}$ конечна, и предположим, что теорема 1 неверна, т.е. функция $e^{c \tau}$ алгебраична над $M(\tau)$. Согласно лемме 1 поле $M(\tau)$ устойчиво под действием группы $\Gamma=S p_{2}(\mathbb{Z})=S L_{2}(\mathbb{Z})$. Поэтому для любого $\gamma \in \Gamma$ функция $e^{c \gamma(\tau)}$ также алгебраична над полем $M(\tau)$. Теперь мы утверждаем, что если $\gamma$ пробегает әруппу $\Gamma$, то функиии $e^{c \gamma(\tau)}$ порождалот над $\mathbb{C}$ поле конечной степени трансиендентности.

Следовательно, все функции $e^{c \gamma(\tau)}$ не могут быть алгебраичными над полем $M(\tau)$ конечной степени трансцендентности, и это противоречие доказьвает теорему 1 в случае $g=1$.

Докажем сформулированное утверждение $(g=1)$. Основное наблюдение заключается в том, что функция $e^{c \tau}$ голоморфна во всей $\tau$-плоскости, а не только в верхней полуплоскости $\mathfrak{H}_{1}$. Поэтому, выбирая элементы $\gamma$ вида $\gamma_{m}=\left(\begin{array}{l}0-1 \\ 1-m\end{array}\right)$, $m=0,1,2, \ldots$, мы получаем, что для каждого $m$ функция $e^{c \gamma_{m}(\tau)}=e^{-c /(\tau-m)}$ голоморфна всюду в $\mathbb{C}$, за исключением сушественно особой точки $\tau=m$. Но любая такая функция $f$ трансцендентна над полем функций, мероморфных в окрестности $m$ (согласно теореме Сохоцкого-Вейершрасса о плотности образа любой проколотой окрестности точки $m$ под действием $f$ ). Следовательно, для каждого положительного целого $t$ функция $e^{c \gamma_{t}(\tau)}$ трансцендентна над полем, порожденньм над $\mathbb{C}$ предыдущими $e^{c \gamma_{m}(\tau)}, m=0,1, \ldots, t-1$, и доказательство утверждения завершается индукцией по $t$.

4.2. Доказательство теоремы 2. Вместо теоремы Акса, как это было в $\S 3$, мы воспользуемся более простым результатом дифференциальной алгебры, полученным Е.Р. Колчиным (см. [7, ч. VI, § 5, упражнение 4(b)]). Этот мультипликативный аналог теоремы Островского следует из того факта, что любая собственная подгруппа группы мультипликативного типа содержится в ядре нетривиального характера.

ПрЕДЛожЕНИЕ 2 (теорема Колчина). Пусть $F \supseteq E \supseteq k \supseteq \mathbb{Q}-$ последовательность полей, $\Delta$-множество дифференцирований поля $F$ таких, что $\delta E \subseteq E$ для всех $\delta \in \Delta, u \bigcap_{\delta \in \Delta} \operatorname{ker} \delta=k$. Пусть әлементы $z_{1}, \ldots, z_{s} \in F^{*}$ алгебраически зависимы над $E$ u $\delta z_{r} / z_{r} \in E$ для всех $\delta \in \Delta u r=1, \ldots, s$. Тогда существуют числа $n_{1}, \ldots, n_{s} \in \mathbb{Z}$, не равнье одновременно нулю, такие, что $z_{1}^{n_{1}} \cdots z_{s}^{n_{s}} \in E^{*}$.

Пусть теперь выполнены все условия теоремы 2, и предположим от противного, что функции $e^{Q_{1}(\boldsymbol{\tau})}, \ldots, e^{Q_{N}(\boldsymbol{\tau})}$ алгебраически зависимы над $M(\boldsymbol{\tau})$. Применяя предложение 2 к $\delta$-дифференциальному полю $E=M(\tau)$ и функциям $z_{r}=e^{Q_{r}(\boldsymbol{\tau})}$ $(r=1, \ldots, s$ c $s:=N)$, мы получаем нетривиальное множество $\left\{c_{1}, \ldots, c_{N}\right\} \in \mathbb{Z}^{N}$ такое, что многочлен

$$
P(\boldsymbol{\tau})=\sum_{1 \leqslant r \leqslant N} c_{r} Q_{r}(\boldsymbol{\tau})
$$

удовлетворяет включению

$$
e^{P(\boldsymbol{\tau})} \in M(\boldsymbol{\tau})
$$


Поскольку многочлены $Q_{r}$ линейно независимы над $\mathbb{Q}$ и не содержат свободные члены, многочлен $P$ ненулевой и тоже без свободных членов, так что согласно лемме 1 для любого $\gamma \in \Gamma$ функция $e^{(\gamma \cdot P)(\boldsymbol{\tau})}=e^{P(\gamma(\boldsymbol{\tau}))}$ также лежит в $M(\boldsymbol{\tau})$.

Рассматривая элементы Г вида

$$
\gamma_{m}=\left(\begin{array}{cc}
\mathbf{0}_{g} & -\mathbf{1}_{g} \\
\mathbf{1}_{g} & -m \mathbf{1}_{g}
\end{array}\right) \in S p_{2 g}(\mathbb{Z}), \quad m=0,1, \ldots,
$$

мы утверждаем, что для любого положительного иелого $t$ функиии $e^{\gamma_{m} \cdot P}$, $m=0, \ldots, t$, алгебраически независимы над $\mathbb{C}$.

Следовательно, для достаточно большого $t$ (более точно, для $t>\frac{1}{2} g(5 g+3)$ в соответствии с формулой (2) из $\S 1)$ все указанные функции не могут лежать в поле $M(\boldsymbol{\tau})$ конечной степени трансцендентности. Полученное противоречие завершает доказательство теоремы 2.

Докажем утверждение $(g \geqslant 1)$. Представляя многочлен $P$ в виде суммы его ненулевых однородных компонент, отвечающих степеням $\geqslant 1$, согласно лемме 3 и факториальности кольца $\mathbb{C}[\tau]$ заключаем, что дивизор в $Z_{g}$ рациональной функции

$$
R_{m}(\boldsymbol{\tau})=\gamma_{m} \cdot P(\tau)=P\left(-\left(\boldsymbol{\tau}-m \mathbf{1}_{g}\right)^{-1}\right), \quad m=0,1, \ldots, t,
$$

совпадает как множество с дивизором $\mathscr{D}_{m}$. Зафиксируем теперь точку $\tau^{\prime} \in \mathscr{D}_{t}$, не принадлежащую полярным дивизорам функций $R_{m}=\gamma_{m} \cdot P, m=0,1, \ldots, t-1$ (что возможно согласно следствию из леммы 2), а также нулевому дивизору функции $R_{t}$ (т.е. отличную от точек неопределенности $R_{t}$ ) и множеству особенностей $\mathscr{D}_{t}$. Тогда функции $e^{R_{m}(\boldsymbol{\tau})}, m=0,1, \ldots, t-1$, лежат в поле $\mathfrak{M}_{\boldsymbol{\tau}^{\prime}}$ ростков мероморфных в $\tau^{\prime}$ функций, и нам остается показать, что функция $f=e^{R_{t}}$ не может удовлетворять нетривиальному алгебраическому уравнению $S(f)=0$ над $\mathfrak{M}_{\boldsymbol{\tau}^{\prime}}$.

Рассмотрим аналитическую кривую $C \subset Z_{g}(\mathbb{C})$, пересекающую $\mathscr{D}_{t}$ трансверсально в точке $\tau^{\prime}$, росток которой в $\tau^{\prime}$ не содержится в нулевых и полярных дивизорах коэффициентов $S$, и обозначим

$$
\phi:\{z \in \mathbb{C}:|z|<1\} \rightarrow C, \quad \phi(0)=\tau^{\prime},
$$

параметризацию $C$. Ввиду трансверсальности и отсутствия неопределенности функции $R_{t}$ в точке $\tau^{\prime}$, функция $f \circ \phi(z)$ одной переменной имеет существенно особую точку в нуле, в то время как прообразы коэффициентов $S$ под действием $\phi$ являются ростками в 0 корректно определенных ненулевых мероморфных функций переменной $z$. Как было отмечено в п. 4.1, получающееся в результате алгебраическое соотношение для $f$ о $\phi$ невозможно, что и завершает доказательство нашего утверждения.

ЗАмЕчАниЕ 2. Применяя предложение 2 дважды, несложно превратить аргументы этого параграфа в чисто алгебраическое доказательство теоремы 2. Действительно, анализ возникает только при доказательстве утверждения. Но если это утверждение неверно, то повторное обращение к теореме Колчина (на этот раз с $E=\mathbb{C}(\boldsymbol{\tau}))$ и тот факт, что ненулевые логарифмические дифференциалы на аффинном пространстве $Z_{g}$ не являются точными, влекут линейную независимость рациональных функций $R_{m}(\boldsymbol{\tau}), m=0,1, \ldots, t$, над $\mathbb{Z} \bmod \mathbb{C}$. Это противоречит следствию из леммы 3 , примененному к многочленам $P_{0}=\cdots=P_{t}:=P$ и $Q=1$. 
ЗАмЕчАНИЕ 3 . Подобным образом при выводе теоремы 1 из теоремы Акса в $\S 3$ мы воспользовались следствием из леммы 3 только для линейных форм $P_{0}, \ldots, P_{t}$ (этот случай допускает более простое доказательство, опирающееся только на лемму 2). Мы оставляем читателю проверку того, что применение следствия из леммы 3 в полном объеме и аргументов из $\S 3$ позволяет доказать теорему 2 . (Необходимо всюду в $\S 3$ заменить $n$ линейных форм $\tau_{j l}$ на $N$ многочленов $Q_{r}$ теоремы 2 , a $\tau_{j l}^{(m)}=\gamma_{m} \cdot \tau_{j l}$ на $\gamma_{m} \cdot Q_{r}$.)

ЗАмечАние 4. Самое главное отличие между доказательствами $\S 3$ и 44 заключается в том, какие именно аргументы из работы [3] мы используем: в $\{3$ нам только необходимо знать, что $M(\boldsymbol{\tau})$ - чисто трансцендентное расширение $M$, в то время как аргументы $\S 4$ основаны исключительно на конечности степени трансцендентности поля $M$ над $\mathbb{C}$. (Разумеется, в обоих случаях нашим основным аргументом является устойчивость поля $M(\tau)$ под действием $\Gamma$.)

\section{§5. Тэта-константы и их логарифмические производные}

Полагая

$$
q_{j l}=e^{2 \pi i \tau_{j l}}, \quad 1 \leqslant j<l \leqslant g, \quad \text { и } \quad q_{j j}=e^{\pi i \tau_{j j}}, \quad 1 \leqslant j \leqslant g,
$$

рассмотрим модулярные формы, именно тәта-константы

$$
\vartheta_{\boldsymbol{a}}=\vartheta_{\left(\boldsymbol{a}^{\prime}, \boldsymbol{a}^{\prime \prime}\right)}(\boldsymbol{q})=\sum_{\boldsymbol{n} \in \mathbb{Z}^{g}} e^{\pi i^{t}\left(\boldsymbol{n}+\boldsymbol{a}^{\prime} / 2\right) \boldsymbol{a}^{\prime \prime}} \prod_{1 \leqslant j \leqslant l \leqslant g} q_{j l}^{\left(n_{j}+a_{j}^{\prime} / 2\right)\left(n_{l}+a_{l}^{\prime} / 2\right)},
$$

отвечающие четным 2-характеристикам $\boldsymbol{a}=\left(\boldsymbol{a}^{\prime}, \boldsymbol{a}^{\prime \prime}\right) \in \mathfrak{K}_{+} \subset(\mathbb{Z} / 2 \mathbb{Z})^{2 g}$, т.е. $\mathrm{c}^{t} \boldsymbol{a}^{\prime} \cdot \boldsymbol{a}^{\prime \prime} \equiv 0(\bmod 2)$. Эти ряды сходятся в некоторой нетривиальной области $\boldsymbol{q}$-пространства, где $\boldsymbol{q}=\left\{q_{j l}, \quad 1 \leqslant j \leqslant l \leqslant g\right\} \in \mathbb{C}^{n}$, но мы будем считать их формальными элементами кольца

$$
\mathfrak{F}_{\boldsymbol{q}}=\mathbb{C}\left[\left[\boldsymbol{q}^{\boldsymbol{\nu}}, 4 \boldsymbol{\nu} \in Z_{g}(\mathbb{Z}), \boldsymbol{\nu} \geqslant 0\right]\right] .
$$

(Для согласования со стандартными разложениями Фурье мы используем обозначение $\boldsymbol{q}^{\boldsymbol{\nu}}=e^{i \pi \operatorname{Tr}(\boldsymbol{\nu} \boldsymbol{\tau})}$.)

Множество частных производных $\boldsymbol{\delta}$ преобразуется теперь в

$$
\delta_{j l}=q_{j l} \frac{\partial}{\partial q_{j l}}, \quad 1 \leqslant j \leqslant l \leqslant g,
$$

и превращает $\mathfrak{F}_{\boldsymbol{q}}$ в дифференциальное кольцо. Определим логарифмические производные тәта-констант формулами

$$
\psi_{\boldsymbol{a}, j l}=\frac{\delta_{j l} \vartheta_{\boldsymbol{a}}}{\vartheta_{\boldsymbol{a}}}, \quad \boldsymbol{a} \in \mathfrak{K}_{+}, \quad 1 \leqslant j \leqslant l \leqslant g .
$$

Поскольку

$$
\vartheta_{\boldsymbol{a}}=1+\sum_{\mathbf{0} \neq \boldsymbol{n} \in \mathbb{Z}^{g}}(-1)^{t^{\prime} \boldsymbol{a}^{\prime \prime}} \prod_{1 \leqslant j \leqslant l \leqslant g} q_{j l}^{n_{j} n_{l}}, \quad \boldsymbol{a}=\left(\boldsymbol{a}^{\prime}, \boldsymbol{a}^{\prime \prime}\right): \boldsymbol{a}^{\prime}=0,
$$


$n$ логарифмических производных, отвечающих $2^{g}$ функциям

$\psi_{\boldsymbol{a}, p q}=\sum_{\mathbf{0} \neq \boldsymbol{n} \in \mathbb{Z}^{g}}(-1)^{t} \boldsymbol{n a}^{\prime \prime} n_{p} n_{q} \prod_{1 \leqslant j \leqslant l \leqslant g} q_{j l}^{n_{j} n_{l}} \cdot \sum_{m=0}^{\infty}\left(-\sum_{\mathbf{0} \neq \boldsymbol{n} \in \mathbb{Z}^{g}}(-1)^{t} \boldsymbol{n} \boldsymbol{a}^{\prime \prime} \prod_{1 \leqslant j \leqslant l \leqslant g} q_{j l}^{n_{j} n_{l}}\right)^{m}$

$(1 \leqslant p \leqslant q \leqslant g)$, лежат в кольце $\mathfrak{F}_{\boldsymbol{q}}$, в то время как логарифмические производные остальных тэта-констант ( $\boldsymbol{a}^{\prime} \neq 0$ ) лежат в поле частных кольца $\mathfrak{F}_{\boldsymbol{q}}$.

Согласно $[3, \S 5]$ поле частных кольца

$$
Q_{g}=\mathbb{Q}\left[\vartheta_{\boldsymbol{a}}, \psi_{\boldsymbol{a}, j l}\right]_{\boldsymbol{a} \in \mathfrak{K}_{+} ; 1 \leqslant j \leqslant l \leqslant g}
$$

является $\boldsymbol{\delta}$-устойчивым и его алгебраическое замыкание совпадает с $M$. Поэтому из теоремы 1 следует

TЕОРЕМА 3. Поле частных кольца

$$
\Pi_{g}=\mathbb{Q}\left[q_{j l}, \vartheta_{\boldsymbol{a}}, \psi_{\boldsymbol{a}, j l}\right]_{\boldsymbol{a} \in \mathfrak{K}_{+} ; 1 \leqslant j \leqslant l \leqslant g}
$$

устойчиво под действием дифферениирований $\delta$ и имеет степень трансцендентности $\frac{1}{2} g(5 g+3)$ над $\mathbb{Q}$.

В классическом случае $g=1$, а также в случае $g=2$ само кольио $Q_{g}$ является устойчивым под действием дифференцирований $\delta$ (см. $[3, \S 6])$; значит, это свойство справедливо также для колец $\Pi_{1}$ и $\Pi_{2}$. Более того, в случае $g=1$ формула произведения для тэта-констант $\vartheta_{a}$ (см., например, $\left.[8, \S 21.42]\right)$ дает явные выражения для $q$-разложений соответствуюших логарифмических производных $\psi_{a}$. Недавние результаты Борчердса показывают, что в этом направлении также существует некоторая аналогия между классическим случаем и $g=2$. Мы подытожим эту статью объяснением того, как с помошью формулы Борчердса выписать явные $\boldsymbol{q}$-разложения десяти элементов, образующих согласно [3, теорема 3 (iv)] базис трансцендентности поля $Q_{2}$ над $\mathbb{Q}$, значит (по теореме 3 ), и поля $\Pi_{2}$ над $\mathbb{Q}[\boldsymbol{q}]$.

Всюду далее мы считаем $g=2$. Как и в $[3, \S 6]$, мы воспользуемся для простоты отображением $(\mathbb{Z} / 2 \mathbb{Z})^{2} \rightarrow\{0,1,2,3\}:$

$$
(0,0) \mapsto 0, \quad(0,1) \mapsto 1, \quad(1,0) \mapsto 2, \quad(1,1) \mapsto 3,
$$

для представления характеристики $\boldsymbol{a}=\left(\boldsymbol{a}^{\prime}, \boldsymbol{a}^{\prime \prime}\right) \in(\mathbb{Z} / 2 \mathbb{Z})^{2} \times(\mathbb{Z} / 2 \mathbb{Z})^{2}$ только двумя цифрами. Тогда $\mathfrak{K}_{+}=\{00,01,02,03,10,12,20,21,30,33\}$. Перенумеруем элементы $\boldsymbol{q}$, полагая $q_{1}=q_{11}, q_{2}=q_{22}, q_{3}=q_{12}$, и проделаем то же самое с дифференцированиями

$$
\delta_{j}=q_{j} \frac{\partial}{\partial q_{j}}, \quad j=1,2,3,
$$

и с логарифмическими производными $\psi_{\boldsymbol{a}, j}$. В этих обозначениях указанньй вьше базис трансцендентности для $Q_{2}$ задается списком

$$
\vartheta_{00}, \vartheta_{01}, \vartheta_{02}, \quad \psi_{00,1}, \psi_{01,1}, \psi_{02,1}, \quad \psi_{00,2}, \psi_{01,2}, \psi_{02,2}, \quad \psi_{00,3}
$$

Наконец, через $Z_{2}^{+}$мы обозначим пространство неотрицательно определенных симметрических матрищ $\boldsymbol{\nu}=\left(\begin{array}{ll}\nu_{1} & \nu_{3} \\ \nu_{3} & \nu_{2}\end{array}\right)$ с элементами $\nu_{1}, \nu_{2}, 2 \nu_{3} \in \mathbb{Z}$, для которых, как и выше, положим $\boldsymbol{q}^{\boldsymbol{\nu}}=q_{1}^{\nu_{1}} q_{2}^{\nu_{2}} q_{3}^{\nu_{3}}$. Тогда выполнено (см. $\left.[9, \S 4]\right)$ 
ПРЕДЛОЖЕНИЕ 3 (формула произведения Борчердса). Справедливо следующее тождество:

$$
\vartheta_{03}(\boldsymbol{q})=\sum_{n_{1}, n_{2} \in \mathbb{Z}}(-1)^{n_{1}+n_{2}} q_{1}^{n_{1}^{2}} q_{2}^{n_{2}^{2}} q_{3}^{n_{1} n_{2}}=\prod_{\mathbf{0}_{2} \neq \boldsymbol{\nu} \in Z_{2}^{+}}\left(\frac{1-\boldsymbol{q}^{\boldsymbol{\nu}}}{1+\boldsymbol{q}^{\boldsymbol{\nu}}}\right)^{f(\operatorname{det} \boldsymbol{\nu})}
$$

əдe

$$
\sum_{m=0}^{\infty} f(m) q^{m}=\left(\sum_{n \in \mathbb{Z}}(-1)^{n} q^{n^{2}}\right)^{-1}=1+2 q+4 q^{2}+8 q^{3}+14 q^{4}+24 q^{5}+\cdots
$$

есть обращение одномерной тәта-константы $\vartheta_{01}(q)$.

Делая подстановки $q_{1} \mapsto-q_{1}$ и $q_{2} \mapsto-q_{2}$ в (5) и (3), находим формулы произведения для трех оставшихся тэта-констант с $\boldsymbol{a}^{\prime}=0$ :

$$
\begin{gathered}
\vartheta_{01}=\prod_{\mathbf{0}_{2} \neq \boldsymbol{\nu} \in Z_{2}^{+}}\left(\frac{1-(-1)^{\nu_{1}} \boldsymbol{q}^{\boldsymbol{\nu}}}{1+(-1)^{\nu_{1}} \boldsymbol{q}^{\boldsymbol{\nu}}}\right)^{f(\operatorname{det} \boldsymbol{\nu})}, \quad \vartheta_{02}=\prod_{\mathbf{0}_{2} \neq \boldsymbol{\nu} \in Z_{2}^{+}}\left(\frac{1-(-1)^{\nu_{2}} \boldsymbol{q}^{\boldsymbol{\nu}}}{1+(-1)^{\nu_{2}} \boldsymbol{q}^{\boldsymbol{\nu}}}\right)^{f(\operatorname{det} \boldsymbol{\nu})} \\
\vartheta_{00}=\prod_{\mathbf{0}_{2} \neq \boldsymbol{\nu} \in Z_{2}^{+}}\left(\frac{1-(-1)^{\nu_{1}+\nu_{2}} \boldsymbol{q}^{\boldsymbol{\nu}}}{1+(-1)^{\nu_{1}+\nu_{2}} \boldsymbol{q}^{\boldsymbol{\nu}}}\right)^{f(\operatorname{det} \boldsymbol{\nu})}
\end{gathered}
$$

Явные формулы для $\boldsymbol{q}$-разложений логарифмических производных этих тэтаконстант очевидным образом следуют из (5), (6). Так, например, в случае $\boldsymbol{a}^{\prime \prime}=3$ и $j=1,2,3$ мы получаем

$$
\begin{aligned}
\psi_{03, j} & =\frac{\delta_{j} \vartheta_{03}}{\vartheta_{03}}=-2 \sum_{\substack{\mathbf{0}_{2} \neq \boldsymbol{\nu} \in Z_{2}^{+} \\
\boldsymbol{0}_{2} \neq \boldsymbol{\mu} \in Z_{2}^{+}}} \frac{\nu_{j} f(\operatorname{det} \boldsymbol{\nu}) \boldsymbol{q}^{\boldsymbol{\nu}}}{1-\boldsymbol{q}^{2 \boldsymbol{\nu}}} \\
& \left.=-2 \boldsymbol{q}_{\substack{\boldsymbol{\mu} \in Z_{2}^{+}:(2 m+1) \boldsymbol{\nu}=\boldsymbol{\mu} \\
m \in \mathbb{Z}, m \geqslant 0}} \nu_{j} f(\operatorname{det} \boldsymbol{\nu})\right) .
\end{aligned}
$$

Тем самым формулы (3) и (6) приводят к обешанным $\boldsymbol{q}$-разложениям всех элементов из нашего списка (4).

ЗАмечАниЕ 5 . В случае $g=2$ разложения в бесконечные произведения существуют и для других тэта-констант, не входящих в изученную выше четверку. В качестве примера отметим тэта-константу $\vartheta_{33}$ (см. [10, пример 2.3], где она названа "максимально нечетной среди четных"). Кроме того, в [10, пример 2.4] приводится разложение в бесконечное произведение модулярной формы веса 5 , заданной формулой $\prod_{a \in \mathfrak{K}_{+}} \vartheta_{\boldsymbol{a}}$. 


\section{Список литературы}

1. Mahler $K$. On algebraic differential equations satisfied by automorphic functions // J. Austral. Math. Soc. 1969. V. 10. P. 445-450.

2. Nishioka K. A conjecture of Mahler on automorphic functions // Arch. Math. (Basel). 1989. V. 53. № 1. P. 46-51.

3. Bertrand D., Zudilin $W$. On the transcendence degree of the differential field generated by Siegel modular forms // Prépubl. de l'Institut de Math. de Jussieu. V. 248, 2000; // http://xxx.lanl.gov/abs/math/0006176.

4. Zudilin $W$. Number theory casting a look at the mirror. Preprint, 2000 (submitted for publication); http://xxx.lanl.gov/abs/math/0008237.

5. Ван дер Варден Б. Л. Алгебра. М.: Наука, 1976.

6. Ax J. On Schanuel's conjectures // Ann. of Math. (2). 1971. V. 93. P. 252-268.

7. Kolchin E. R. Differential algebra and algebraic groups. Pure Appl. Math. V. 54. N. Y.-London: Academic Press, 1973.

8. Уиттекер Э.Т., Ватсон Дж. Н. Курс современного анализа. Т. 2. М.: Физматлит, 1963.

9. Kontsevich $M$. Product formulas for modular forms on $O(2, n)$ (after R. Borcherds) [Exp. no. 821] // Astérisque 1997. V. 245. P. 41-56; // Sém. Bourbaki. V. 1996/97. Exp. 820-834, http://xxx.lanl.gov/abs/alg-geom/9709006.

10. Gritsenko V.A., Nikulin V. V. Automorphic forms and Lorentzian Kac-Moody algebras. Part II // Internat. J. Math. 1998. V. 9. № 2. P. 201-275.

(Д. Бертран) Institut de Mathématiques de Jussieu, Paris (В. В. Зудилин) Московский государственный университет

Поступило в редакцию им. М. В. Ломоносова

26.XII. 2000

E-mail: bertrand@math.jussieu.fr, wadim@ips.ras.ru 